\title{
Vigilancia de laboratorio de enfermedad meningocóccica invasora en Chile, 2006-2012
}

\author{
Pamela Araya, Janepsy Díaz, Mabel Seoane, Jorge Fernández, Solana Terrazas, Andrea Canals, \\ Alejandra Vaquero, Gisselle Barra, Juan C. Hormazábal, Paola Pidal y M. Teresa Valenzuela
}

\section{Laboratory surveillance for invasive meningococcal disease in Chile, 2006-2012}

Background: Laboratory surveillance of Invasive Meningococcal Disease (IMD) is performed by the Institute of Public Health of Chile. It confirms identification, classifies in serogroups and analyzes the genetic profiles of Neisseria meningitidis isolates from laboratories throughout the country. Aim: To show the results of this surveillance from 2006 to 2012. Methods: A descriptive data analysis of the confirmed cases of IMD and serological characterization, susceptibility and genetic profiles of the isolates. The analysis was disaggregated by serogroup, age and region. Results: From 2006 to 2012, 486 isolates of $N$. meningitidis were confirmed. In 2011 a rise in IMD rates was observed due to an increase in W serogroup cases, mainly affecting children aged 5 years or less. Serogroup W became the most prevalent during 2012 (58.3\%), replacing the historically prevalent serogroup B. Predominating strains belonged to ST-32 complex/ET-5 complex (40, 4\% of strains) and ST-41/44 complex/ Lineage 3 (45, $9 \%$ of strains). Conclusions: Laboratory surveillance has allowed the early detection of increasing IMD caused by serogroup $\mathrm{W}$, which is emergent in Chile. This information has reinforced the daily monitoring of new cases, in collaboration with all the clinical laboratories of the country.

Key words: Meningococcal invasive disease, surveillance, Neisseria meningitidis, Serogroup W.

Palabras clave: Enfermedad meningocóccica invasora, vigilancia, Neisseria meningitidis, meningococo serogrupo $\mathrm{W}$.

\section{Introducción}

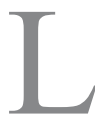

a enfermedad meningocóccica invasora (EMI) causada por Neisseria meningitidis continúa siendo un problema de salud pública de distribución mundial, debido a su potencial epidémico y su alta morbi-mortalidad a corto y largo plazo ${ }^{1-5}$. La EMI se presenta en la mayoría de los casos como meningitis o como meningococcemia. Es una enfermedad que puede dejar secuelas, alterando la calidad de vida de los que la padecen y tiene una letalidad de alrededor de $10 \%$.

Neisseria meningitidis es un diplococo gramnegativo, con 12 serogrupos identificados ${ }^{7}$. De ellos, A, B, C, Y, $\mathrm{W}$ y X son los más frecuentes y se han identificado en importantes brotes en los últimos años ${ }^{8}$. Los serogrupos $\mathrm{B}$ y $\mathrm{C}$ son los de mayor ocurrencia en el mundo, sobre todo en Europa y América. En África predomina el serogrupo A, pero también se han reportado brotes por los serogrupos W y X y en Asia predominan los serogrupos A y $\mathrm{C}^{9}$. En el último tiempo se ha observado un aumento en la incidencia del serogrupo $\mathrm{Y}$ en Estados Unidos de América (E.U.A.), Suecia e Israel ${ }^{10-12}$.

La presentación de esta enfermedad como endemia y como brotes ha permitido observar su comportamiento epidemiológico, así como ha motivado el desarrollo de nuevas tecnologías para la prevención, diagnóstico y tratamiento de la enfermedad ${ }^{8,13}$. Se han descrito importantes brotes durante la Primera y Segunda Guerra Mundial ${ }^{8,14}$ y durante las peregrinaciones religiosas de musulmanes a la zona de La Meca ${ }^{15,16}$, las que causaron preocupación debido a que favorecieron la diseminación del brote a otras regiones, e incluso otros continentes, identificándose un aumento de casos por nuevos serogrupos en varios países. Así se dio explicación al cambio en la epidemiología en los brotes ocurridos en E.U.A., donde se presentaron casos de EMI por el serogrupo $\mathrm{W}$ en peregrinos que regresaron del Hajj en La Meca, Arabia Saudita ${ }^{15,17-21}$. Las causas de estas epidemias aún no han sido completamente definidas. Sin embargo, su rápida diseminación se ha asociado a factores como el hacinamiento ${ }^{22-24}$, el desplazamiento de poblaciones ${ }^{25}$, factores climáticos ${ }^{26}$ y la virulencia de las cepas circulantes ${ }^{27}$.

Durante los últimos años se ha observado un aumento de los casos de EMI causados por los serogrupos Y y W en varias regiones del mundo y en América Latina ${ }^{28,29}$.

En Chile, se ha mantenido una vigilancia del comportamiento de $N$. meningitidis desde 1976, donde se registraba un predominio endémico del serogrupo C (90\% de los casos) ${ }^{30}$. Sin embargo, hacia 1990 se presentó un aumento en los casos por el serogrupo B, convirtiéndose
Instituto de Salud Pública de Chile.

Departamento de Laboratorio Biomédico Nacional y de Referencia Sub-departamento de

Enfermedades Infecciosas (PA, MS, JCH, PP).

Subdepartamento de Genética

Molecular (JF, GB).

Departamento de Asuntos

Científicos (JD, ST, AC, AV).

Dirección (MTV)

Este artículo se realizó con información recopilada por el Laboratorio Biomédico Nacional de Referencia del Instituto de Salud Pública de Chile, sin financiamiento externo.

Los autores de este artículo declaran no tener conflictos de interés.

Recibido: 10 de diciembre de 2013 Aceptado: 7 de abril de 2014

\section{Correspondencia a:}

Janepsy Díaz Tito

jdiaz@ispch.cl 
en el responsable de cerca de $80 \%$ de los casos de EMI. Esta vigilancia permitió la detección de brotes y cambios de serogrupos causantes de EMI en la población, como por ejemplo lo sucedido en 1994 cuando la EMI por serogrupo $\mathrm{C}$ se consideró una enfermedad re-emergente en Chile ${ }^{31}$ y la detección de los brotes de los años 2000 y 2002, lo cual permitió la implementación temprana de una campaña de vacunación para su control ${ }^{32}$. La vigilancia universal e inmediata de la enfermedad meningocóccica se estableció en Chile el año $2004^{33}$ y tiene como objetivo la detección temprana de aumento de casos y la toma precoz de medidas de control.

La vigilancia de laboratorio es parte de la vigilancia universal y se realiza en el Laboratorio Biomédico Nacional y de Referencia del Instituto de Salud Pública de Chile (ISP), encargado de la confirmación de las muestras de casos sospechosos provenientes desde los laboratorios clínicos públicos y privados del país. Una vez confirmadas como $N$. meningitidis, se realiza la seroagrupación, el estudio de susceptibilidad antimicrobiana y el estudio genético $^{33}$. El objetivo de esta publicación es dar a conocer los resultados de la vigilancia de laboratorio desde el año 2006 a 2012 en Chile.

\section{Materiales y Métodos}

Se realizó un análisis descriptivo de los datos registrados por el Laboratorio Biomédico Nacional y de Referencia del ISP durante los años 2006 a 2012. Toda la información fue registrada en bases de datos, asegurando que cada cepa reportada perteneciera a un caso único. Se realizó un análisis descriptivo de los casos confirmados de enfermedad por $N$. meningitidis, los cuales se desagregaron por año, edad y región. Los análisis de resultados fueron procesados con el software estadístico Stata 11.0.

\section{Vigilancia de laboratorio}

Las cepas identificadas como $N$. meningitidis por los laboratorios clínicos públicos y privados del país son derivados al Laboratorio de Meningitis Bacteriana-Sección Bacteriología del ISP, donde se realiza la confirmación de este agente, la caracterización serológica y el análisis de susceptibilidad antimicrobiana según protocolos internacionales de vigilancia de laboratorio y de la Red SIREVA de la Organización Panamericana de la Salud ${ }^{34,35}$ Se define caso confirmado de EMI como aquel en el cual se logra el aislamiento de $N$. meningitidis a partir del cultivo de líquido cefalorraquídeo (LCR), sangre u otro fluido o tejido de sitio estéril, o se confirma la presencia de $N$. meningitidis por técnicas de reacción de polimerasa en cadena (RPC) en tiempo real desde el LCR o plasma. Los casos sospechosos que tengan nexos epidemiológicos con casos confirmados, también se consideran casos confirmados $^{36}$.

\section{Susceptibilidad in vitro}

Todas las cepas confirmadas son sometidas a análisis de susceptibilidad in vitro, para penicilina, ceftriaxona, cloranfericol, rifampicina y ciprofloxacina. Se determinó la concentración inhibitoria mínima (CIM) mediante el método de microdilución en caldo y epsilometría según los estándares del Clinical and Laboratory Standards Institute vigente (CLSI) ${ }^{37}$. En este informe se reportan las susceptibilidades obtenidas para las cepas confirmadas durante el período 2010-2012.

\section{Análisis genético}

El estudio del subtipo genético se realizó con electroforesis en gel de campo pulsado (EGCP) para establecer relaciones filogenéticas ${ }^{38,39}$, y con secuenciamiento de multilocus (en inglés multilocus sequence typing-MLST) que permite identificar y diferenciar los distintos linajes genéticos que componen la estructura poblacional en una base de datos (http://pubmlst.org/). En N. meningitidis se analizan 7 locis: abcZ, adk, aroE, fumC, gdh, pdh y pgm $^{40}$. Se caracterizó un total de 223 cepas correspondientes a los años 2010 a 2012.

\section{Resultados}

En el período 2006-2012 fue confirmado un total de 507 cepas de $N$. meningitidis en el ISP. Entre los años 2006 y 2010 se observó una disminución en el número de casos confirmados y en las tasas de EMI en la población (valor p para tendencia: 0,01). Sin embargo, esta tendencia se revertió a partir del año 2011, observándose un aumento significativo (valor p para tendencia: $<0,001$ ) (Figura 1).

Del total de casos confirmados durante este período, se obtuvo el dato de procedencia de 486 casos. El 52,1\% provenían de la Región Metropolitana (RM) y le seguían en frecuencia las regiones del Bío-Bío y de Valparaíso con porcentajes de 11,9 y $10,3 \%$, respectivamente. Durante el período, se observó una disminución de las tasas de EMI en la zona centro sur del país, la cual tuvo un leve aumento durante el 2012. Por otro lado, se observó un aumento de las tasas de EMI principalmente en la RM y en la zona centro norte (Figura 2). Sin embargo, al comparar los serogrupos de los casos confirmados por región, se observó que el aumento de la tasa de casos de EMI en la RM estuvo dado principalmente por el aumento del serogrupo W, mientras que en las zonas Centro Norte y Centro Sur, el aumento de los casos confirmados se debió al serogrupo B (Tabla 1).

El 50,9\% de las cepas confirmadas de $N$. meningitidis en el período 2006-2012 correspondía a niños bajo 5 años de edad. En este grupo, las tasas fueron más altas que en el resto de la población durante todo el período, presentando un incremento a partir del año 2010. La distribución de 


\begin{tabular}{|llc|}
\multicolumn{3}{c}{ Tabla 1. Frecuencia de casos de serogrupos B y W por } \\
zona, año 2012
\end{tabular}

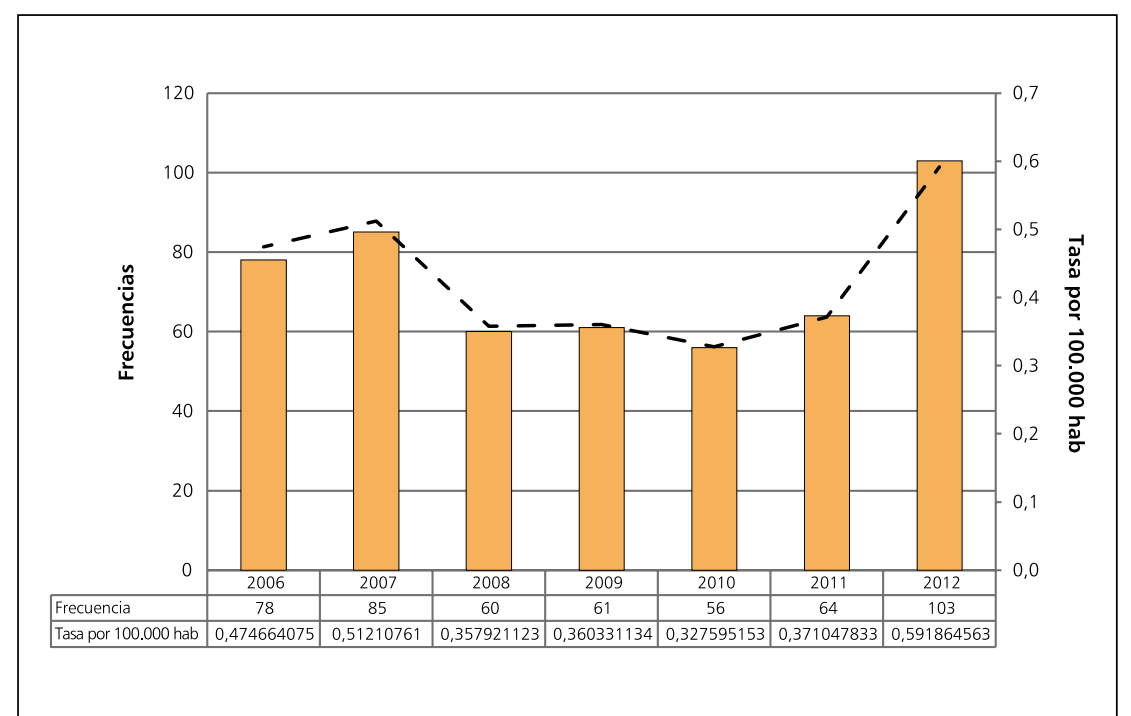

Figura 1. Número y tasas por 100.000 habitantes de casos confirmados de enfermedad invasora por Neisseria meningitidis, por año. Chile, 2006-2012.

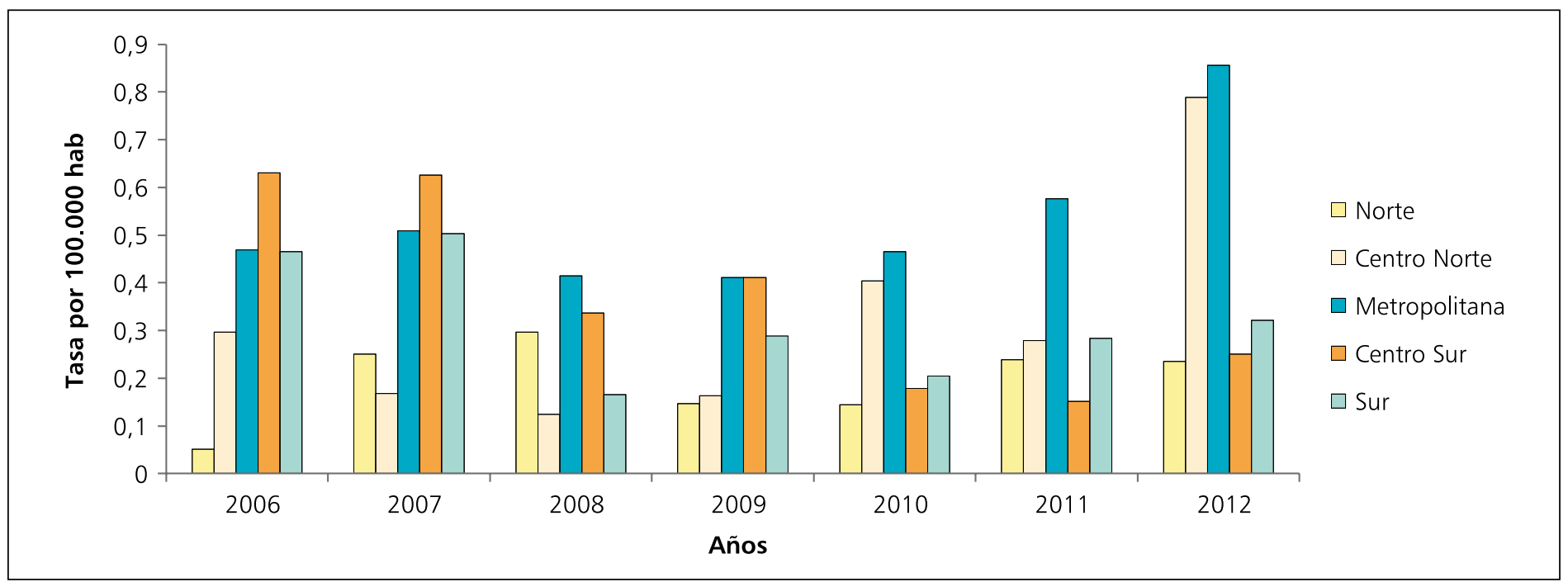

Figura 2. Distribución regional de los casos confirmados de enfermedad invasora por Neisseria meningitidis en Chile, período $2006-2012$.

las tasas de casos confirmados de $N$. meningitidis en los niños de 4 años o más fue similar en los distintos años del período, registrándose un aumento en la tasa el año 2012 (Figura 3).

Las tasas de incidencia de casos confirmados de $N$. meningitidis, reveló que el serogrupo B predominó en la población durante el período 2006-2012, aun registrándose un descenso significativo en las tasas durante el período (valor $\mathrm{p}$ para tendencia: $<0,001$ ). A pesar del marcado descenso en la incidencia de casos confirmados de serogrupo B, se observó una pequeña alza en la tasa en año 2012. Por otro lado, el serogrupo W presentó una alza significativa en su tasa de incidencia anual a partir del año 2010 (valor p para tendencia: $<0,001$ ), alcanzando a ser el serogrupo con mayor incidencia en la población durante el año 2012 (Figura 4).

El total de casos confirmados de $N$. meningitidis en el período 2010-2012 fue sometido a análisis de susceptibilidad in vitro. Todos los casos resultaron sensibles a ceftriaxona, cloranfenicol, rifampicina y ciprofloxacina. Se encontró un mayor porcentaje de casos de sensibilidad intermedia a penicilina en los años 2010 y 2011, lo 
Figura 3. Tasa específica por grupos de edad de casos confirmados de Neisseria meningitidis, por año.

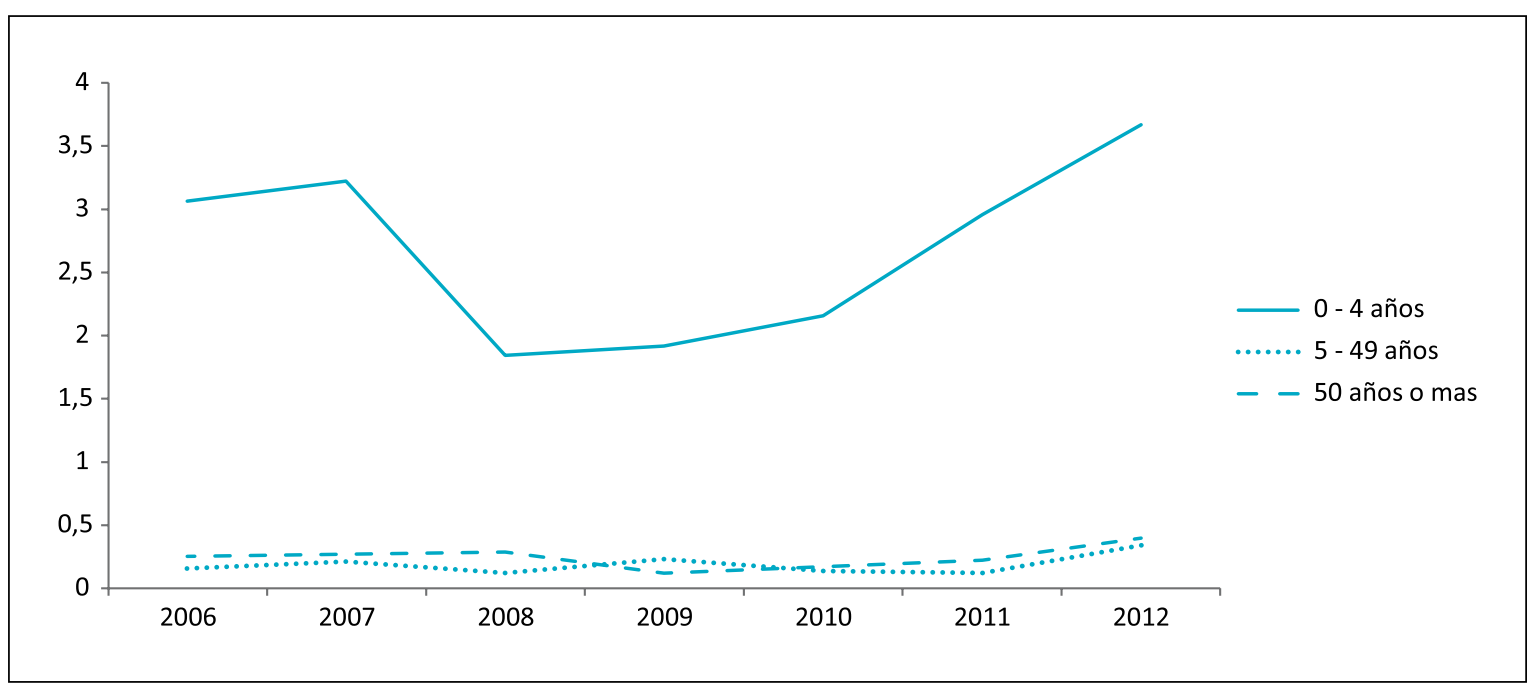

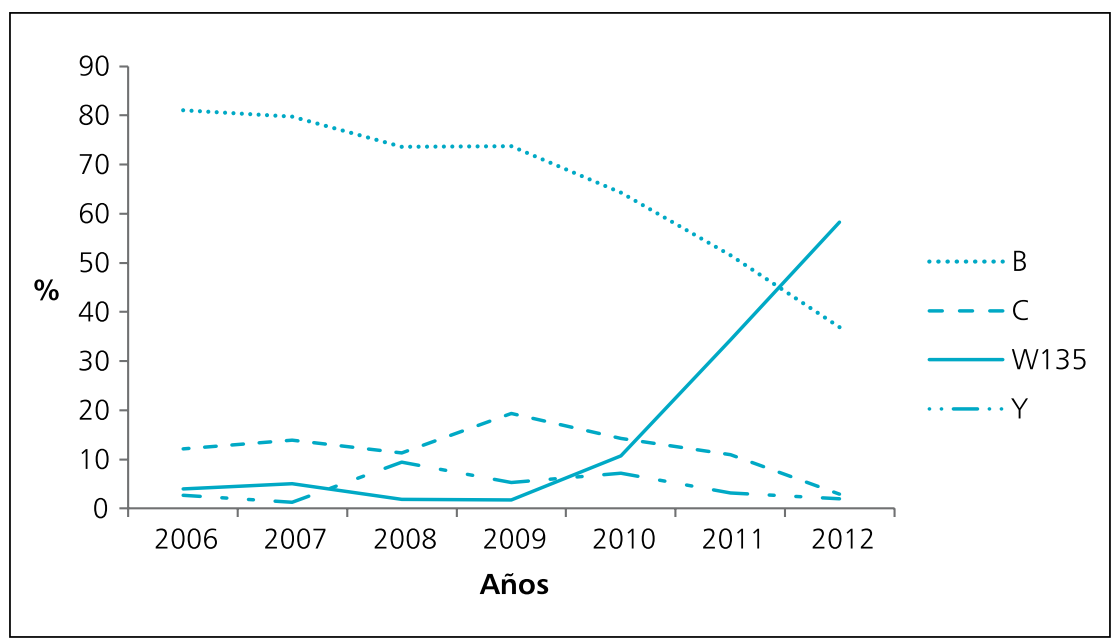

Figura 4. Porcentaje de casos confirmados de Neisseria meningitidis, por serogrupo y por año. cual se revirtió para el año 2012. Las cepas confirmadas como serogrupo $\mathrm{W}$ presentaron una mayor sensibilidad a penicilina (Tabla 2).

El análisis genético de las cepas serogrupo B reveló que éstas tenían una distribución heterogénea en las clasificaciones con EGCP, sin destacar algún grupo en particular. Sin embargo, al analizar los complejos clonales, predominaron principalmente las cepas pertenecientes al complejo clonal ST-32 complex/ET-5 complex (40,4\% de las muestras) y el ST-41/44 complex/Lineage 3 (45,9\% de las muestras).

Por otro lado, las cepas del serogrupo $\mathrm{W}$ revelaron un predominio del grupo Cl-Nm-Spe-031 con una marcada tendencia al aumento el año 2012. Este grupo pertenece al complejo clonal ST-11 complex/ET 37 complex, el que se identificó en $95,5 \%$ de las muestras analizadas (Tabla 3).

Tabla 2. Análisis de susceptibilidad del total cepas confirmadas de Neisseria meningitidis y de cepas confirmadas como serogrupo W, por año, 2010-2012

\begin{tabular}{|c|c|c|c|c|c|c|c|}
\hline \multirow[t]{2}{*}{ Año } & \multirow[t]{2}{*}{$n$} & \multicolumn{2}{|c|}{ Penicilina } & \multirow{2}{*}{$\begin{array}{c}\text { Ceftriaxona } \\
\text { S (\%) }\end{array}$} & \multirow{2}{*}{$\begin{array}{c}\text { Cloranfenicol } \\
\text { S (\%) }\end{array}$} & \multirow{2}{*}{$\begin{array}{l}\text { Rifampicina } \\
\text { S (\%) }\end{array}$} & \multirow{2}{*}{$\begin{array}{c}\text { Ciprofloxacina } \\
\text { S (\%) }\end{array}$} \\
\hline & & S (\%) & I (\%) & & & & \\
\hline \multicolumn{8}{|c|}{ Total de cepas } \\
\hline 2010 & 56 & 19,6 & 80,4 & 100 & 100 & 100 & - \\
\hline 2011 & 64 & 31,3 & 68,8 & 100 & 100 & 100 & 100 \\
\hline 2012 & 103 & 55,3 & 44,7 & 100 & 100 & 100 & 100 \\
\hline \multicolumn{8}{|c|}{ Serogrupo W } \\
\hline 2010 & 6 & 50,0 & 50,0 & 100 & 100 & 100 & - \\
\hline 2011 & 22 & 77,3 & 22,7 & 100 & 100 & 100 & 100 \\
\hline 2012 & 60 & 82,0 & 18,0 & 100 & 100 & 100 & 100 \\
\hline
\end{tabular}




\begin{tabular}{|c|c|c|c|c|c|c|}
\hline Serogrupo & Complejo clonal & Tipo de secuencia & PFGE & $\begin{array}{c}2010 \\
(n)\end{array}$ & $\begin{array}{c}2011 \\
(n)\end{array}$ & $\begin{array}{c}2012 \\
(n)\end{array}$ \\
\hline \multirow[t]{2}{*}{$\begin{array}{l}\text { B } \\
(n: 109)\end{array}$} & $\begin{array}{l}\text { ST-32 complex/ET-5 complex } \\
\text { (n: 44) }\end{array}$ & 32 (n: 28) & $\begin{array}{l}\text { Cl-Nm-Spe-001 } \\
\text { Cl-Nm-Spe-047 }\end{array}$ & $\begin{array}{l}5 \\
0\end{array}$ & $\begin{array}{l}0 \\
1\end{array}$ & $\begin{array}{l}3 \\
3\end{array}$ \\
\hline & $\begin{array}{l}\text { ST-41/44 complex/Lineage } 3 \\
\text { (n: 50) }\end{array}$ & 44 (n: 35) & $\begin{array}{l}\text { Cl-Nm-Spe-014 } \\
\text { Cl-Nm-Spe-082 }\end{array}$ & $\begin{array}{l}2 \\
3\end{array}$ & $\begin{array}{l}1 \\
2\end{array}$ & $\begin{array}{l}2 \\
2\end{array}$ \\
\hline \multirow[t]{2}{*}{$\begin{array}{l}\text { W } \\
(\mathrm{n}: 88)\end{array}$} & $\begin{array}{l}\text { ST-11 complex/ET } 37 \text { complex } \\
\text { (n: 84) }\end{array}$ & 11 (n: 67) & $\begin{array}{l}\text { Cl-Nm-Spe-030 } \\
\text { Cl-Nm-Spe-031 } \\
\text { Cl-Nm-Spe-046 }\end{array}$ & $\begin{array}{l}2 \\
3 \\
0\end{array}$ & $\begin{array}{l}6 \\
1 \\
3\end{array}$ & $\begin{array}{r}7 \\
26 \\
7\end{array}$ \\
\hline & & 1.025 (n: 11) & Cl-Nm-Spe-031 & 0 & 4 & 7 \\
\hline
\end{tabular}

\section{Discusión}

El sistema de vigilancia de laboratorio de $N$. meningitidis que realiza el ISP de Chile, permitió una temprana detección del aumento de casos de EMI y de la emergencia del serogrupo $\mathrm{W}^{41}$. Al igual que otros países de América Latina, Chile ha presentado un aumento sostenido, tanto en los casos de EMI por W como en la letalidad de las EMI en los últimos tres años. En Brasil, Argentina y Uruguay se ha descrito un aumento de los casos por el serogrupo W a partir del año 2002 ${ }^{29}$. En Argentina se registró un incremento el año $2006^{42}$, alcanzando a $49 \%$ de los casos de EMI en el año 2010 y el serogrupo aislado con mayor frecuencia el año $2011^{43}$. Brasil ha presentado gran variabilidad en el serogrupo prevalente; sin embargo, también ha registrado un alza en los casos por el serogrupo $\mathrm{W}$, alcanzando a 5,6\% de los aislados analizados ${ }^{35}$.

El mayor aumento de las tasas de EMI se registró en la RM, lo cual podría estar explicado por la mayor densidad poblacional que presenta la región, con respecto a las otras regiones, facilitando la transmisión persona-persona del agente etiológico ${ }^{44}$. Por otro lado, Santiago, la capital de Chile y de la RM, es el punto de llegada y de salida de inmigrantes y turistas, posibilitando la importación de nuevos agentes infecciosos y su posterior transmisión, como sucedió con el virus influenza pandémico $\mathrm{A}(\mathrm{H} 1 \mathrm{~N} 1)$ en el año $2009^{45,46}$. La segunda región que presentó aumento en sus tasas de EMI fue la Región de Valparaíso. No obstante de su importante actividad turística y portuaria, el aumento de casos de EMI por $N$. meningitidis se dio principalmente por el aumento de casos serogrupo B y no $\mathrm{W}$, lo cual sugiere que este aumento de casos estaría dado por un fenómeno diferente al antes mencionado.

El mayor porcentaje de casos se presentó en niños bajo cinco años, principalmente en los lactantes menores de un año. La campaña de vacunación implementada por el Ministerio de Salud, ha logrado disminuir los casos de EMI por W a cero en lactantes entre 9 meses y 5 años de edad, vacunados hasta octubre de $2013^{47,48}$. El aumento en el número de casos por $\mathrm{W}$, ha llevado a que este serogrupo sea la causa más importante de EMI en Chile, desplazando al serogrupo B, tendencia que ha continuado durante el $2013^{47,48}$. Esta evolución en la epidemiología de las EMI ha sido reportado en América Latina ${ }^{49}$, donde las principales cepas identificadas en los brotes de EMI han variado en el tiempo. Este cambio también se ha observado en África ${ }^{2}$, donde la vacunación contra $N$. meningitidis serogrupo A provocó un aumento de casos dado por el serogrupo W. Una situación similar ocurrió en China ${ }^{50}$, donde se reportó un brote por $N$. meningitidis serogrupo $\mathrm{C}$, generando un cambio en la epidemiología del serogrupo y en la edad de los casos, porque afectó principalmente a adolescentes y adultos.

La sensibilidad in vitro intermedia a penicilina se ha identificado en Chile hace ya algunos años ${ }^{51-54}$. Aunque no se registró resistencia total a este antimicrobiano, se observó un aumento en la sensibilidad intermedia en el serogrupo B, no así en el serogrupo W. Este patrón de susceptibilidad se presenta en otros países de América Latina, donde predomina la sensibilidad total o intermedia a penicilina en las cepas analizadas y sensibilidad total a ciprofloxacina, rifampicina y cloranfenicol ${ }^{55-57}$.

El análisis genético de las cepas reveló que el complejo clonal ST-11 es el predominante en el serogrupo W, como ya se había reportado anteriormente ${ }^{58}$. Este complejo se relaciona con los clones identificados en la epidemia ocurrida durante la peregrinación a La Meca en los años 2000-2001, clon que se exportó a diferentes países ${ }^{59-61}$. Este clon también ha sido identificado como un clon hipervirulento, lo cual coincide con lo sucedido en Chile, donde los casos de EMI se han manifestado como cuadros graves, principalmente meningococcemias, y con una alta letalidad ${ }^{41,62}$.

La EMI por serogrupo W es una enfermedad nueva en Chile, la que ha ido en aumento, cambiando el escenario epidemiológico nacional. El sistema de notificación obligatoria inmediata ha permitido detectar tempranamente el incremento de casos de EMI y la vigilancia de laboratorio 
ha permitido la identificación del serogrupo $\mathrm{W}$, emergente en Chile, facilitando la implementación temprana de medidas de prevención como la vacunación a niños entre 9 meses y cinco años y el adecuado control de posibles contactos de los casos confirmados. La información proporcionada por el sistema de vigilancia nos ha obligado a estar en permanente alerta y monitoreo de casos diarios, mediante la participación activa de todos los laboratorios clínicos del país, así como en la difusión de las medidas de prevención y control.

\section{Resumen}

Introducción: La vigilancia de laboratorio de enfermedad meningocócica invasora (EMI) que realiza el Instituto de Salud Pública de Chile, confirma, seroagrupa y estudia el perfil genético de las cepas de Neisseria meningitidis provenientes de los laboratorios del país. Objetivo: En este artículo se muestra los resultados de esta vigilancia entre los años 2006 a 2012. Materiales y
Métodos: Se realizó un análisis descriptivo de los casos confirmados de EMI, caracterización serológica, el análisis de susceptibilidad antimicrobiana y el estudio de subtipo genético de la cepa. El análisis se desagregó por serogrupo, edad y región. Resultados: En el período 2006-2012 fue confirmado un total de 486 cepas de $N$. meningitidis. A partir del año 2011 se observó un alza en la tasa de EMI dado por el número de casos del serogrupo $\mathrm{W}$, afectando principalmente a niños bajo 5 años de edad. El W se transformó en el serogrupo prevalente el año 2012 (58,3\%), desplazando al serogrupo B, el cual históricamente había sido prevalente. Predominaron principalmente las cepas pertenecientes al complejo clonal ST-32 complex/ET-5 complex $(40,4 \%$ de las muestras) y el ST-41/44 complex/Lineage 3 (45,9\% de las muestras). Conclusiones: El sistema de vigilancia de laboratorio ha permitido la identificación del serogrupo W, emergente en Chile. Esta información nos ha obligado a estar en permanente alerta y monitoreo de casos diarios, mediante la participación activa de todos los laboratorios clínicos del país.

\section{Referencias bibliográficas}

1.- Davie S, Glennie L, Rowland K. Towards a meningitis free world-can we eliminate meningococcal meningitis?: contribution of the meningitis patient groups. Vaccine 2012; 30 Suppl 2: B98-B105.

2.- Halperin S A, Bettinger J A, Greenwood B, Harrison L H, Jelfs J, Ladhani S N, et al. The changing and dynamic epidemiology of meningococcal disease. Vaccine 2012; 30 Suppl 2: B26-36.

3.- Jordens J Z, Williams J N, Jones G R, Heckels J E. Detection of meningococcal carriage by culture and PCR of throat swabs and mouth gargles. J Clin Microbiol 2002; 40 (1): 75-9.

4.- Judelsohn R, Marshall G S. The burden of infant meningococcal disease in the United States. $\mathrm{J}$ Pediatric Infect Dis Soc 2012; 1 (1): 64-73.

5.- Maiden M C, Frosch M. Can we, should we, eradicate the meningococcus? Vaccine 2012; 30 Suppl 2: B52-6.

6.- Campsall P A, Laupland K B, Niven D J. Severe meningococcal infection: a review of epidemiology, diagnosis, and management. Crit Care Clin 2013; 29 (3): 393-409.

7.- Harrison O B, Claus H, Jiang Y, Bennett J S, Bratcher H B, Jolley K A, et al. Description and nomenclature of Neisseria meningitidis capsule locus. Emerg Infect Dis 2013; 19 (4): 566-73.

8.- World Health Organization Emerging and other Communicable Diseases, Surveillance and Control. Control of epidemic meningococcal disease. WHO practical guidelines. 2 nd ed.
Geneve, Switzerland: WHO; 1998.

9.- Rosenstein N E, Perkins B A, Stephens D S, Popovic T, Hughes J M. Meningococcal disease. N Engl J Med 2001; 344 (18): 1378-88.

10.- Centers for Disease Control and Prevention (CDC). Serogroup Y meningococcal diseaseIllinois, Connecticut, and selected areas, United States, 1989-1996. MMWR Morb Mortal Wkly Rep 1996; 45 (46): 1010-3.

11.- Hedberg S T, Toros B, Fredlund H, Olcen P, Molling P. Genetic characterisation of the emerging invasive Neisseria meningitidis serogroup Y in Sweden, 2000 to 2010. Euro Surveill 2011; 16 (23): 19885 .

12.- Whitney A M, Coulson G B, von Gottberg A, Block C, Keller N, Mayer L W, et al. Genotypic comparison of invasive Neisseria meningitidis serogroup Y isolates from the United States, South Africa, and Israel, isolated from 1999 through 2002. J Clin Microbiol 2009; 47 (9): 2787-93.

13.- Harrison L H, Pelton S I, Wilder-Smith A, Holst J, Safadi M A, Vazquez J A, et al. The Global Meningococcal Initiative: recommendations for reducing the global burden of meningococcal disease. Vaccine 2011; 29 (18): 3363-71.

14.- Pollard A J. Global epidemiology of meningococcal disease and vaccine efficacy. Pediatr Infect Dis J 2004; 23 (12 Suppl): S2749.

15.- Lingappa J R, Al-Rabeah A M, Hajjeh R, Mustafa T, Fatani A, Al-Bassam T, et al. Serogroup W-135 meningococcal disease during the Hajj, 2000. Emerg Infect Dis 2003;9 (6):
665-71.

16.- Kelly D, Pollard A J. W135 in Africa: origins, problems and perspectives. Travel Med Infect Dis 2003; 1 (1): 19-28.

17.- Wilder-Smith A, Goh KT, Barkham T, Paton N I. Hajj-associated outbreak strain of Neisseria meningitidis serogroup W135: estimates of the attack rate in a defined population and the risk of invasive disease developing in carriers. Clin Infect Dis 2003; 36 (6): 679-83.

18.- Yamamoto K, Kato Y, Shindo T, Ujiie M, Takeshita N, Kanagawa S, et al. Meningococcemia due to the 2000 Hajjassociated outbreak strain (serogroup W-135 ST-11) with immunoreactive complications. Jpn J Infect Dis 2013; 66 (5): 443-5.

19.- Doganci L, Baysallar M, Saracli M A, Hascelik G, Pahsa A. Neisseria meningitidis W135, Turkey. Emerg Infect Dis 2004; 10 (5): 936-7.

20.- Aguilera J F, Perrocheau A, Meffre C, Hahne S, W135 Working Group. Outbreak of serogroup W135 meningococcal disease after the Hajj pilgrimage, Europe, 2000. Emerg Infect Dis 2002; 8 (8): 761-7.

21.- Centers for Disease Control and Prevention (CDC). Risk for meningococcal disease associated with the Hajj 2001. MMWR Morb Mortal Wkly Rep 2001; 50 (6): 97-8.

22.- Fone D L, Harries J M, Lester N, Nehaul L. Meningococcal disease and social deprivation: a small area geographical study in Gwent, UK. Epidemiol Infect 2003; 130 (1): 53-8.

23.- Stuart J M, Middleton N, Gunnell D J. 
Socioeconomic inequality and meningococcal disease. Commun Dis Public Health 2002; 5 (4): 327-8.

24.- Williams C J, Willocks L J, Lake I R, Hunter P R. Geographic correlation between deprivation and risk of meningococcal disease: an ecological study. BMC Public Health 2004; 4: 30 .

25.- Stephens D S. Uncloaking the meningococcus: dynamics of carriage and disease. Lancet 1999; 353 (9157): 941-2.

26.- Tzeng Y L, Stephens D S. Epidemiology and pathogenesis of Neisseria meningitidis. Microbes Infect 2000; 2 (6): 687-700.

27.- Buckee C O, Jolley K A, Recker M, Penman B, Kriz P, Gupta S, et al. Role of selection in the emergence of lineages and the evolution of virulence in Neisseria meningitidis. Proc Natl Acad Sci U S A 2008; 105 (39): 15082-7.

28.- Nelson J D. Jails, microbes, and the three-foot barrier. N Engl J Med 1996; 335 (12): 885-6.

29.- López E L, Debbag R. Meningococcal disease: always present. Serogroup changes in the Southern Cone. Rev Chilena Infectol 2012; 29 (6): 587-94.

30.- García J, Prat M, Toro J. Meningitis meningocócica en la ciudad de Santiago a dos años de la vacunación masiva. Evaluación preliminar. Bol Inst Salud Pública Chile 1980; XXI $(1,2)$.

31.- Gallegos D, Maldonado A, Cáceres K, Seoane M. Situación epidemiológica y cumplimiento de indicadores de la vigilancia de la enfermedad meningocócica. El Vigía, Boletín de Vigilancia en Salud Pública, 2012; 13 (27): 59-63.

32.- Werner M, Sepúlveda P, Gutiérrez A. Segundo bloqueo epidemiológico enfermedad menigocócica serogrupo $\mathrm{C}$ en el Servicio de Salud Concepción, 2002. El Vigía, Boletín de Vigilancia en Salud Pública de Chile 2002 Jul/ Dic; 6 (17): 37.

33.- Ministerio de Salud. Reglamento sobre Notificación de Enfermedades Transmisibles de Declaración Obligatoria. Diario Oficial 2005, 10 Mayo; Decreto Supremo No 158/04.

34.- Ministerio de Salud. Instituto de Salud Pública de Chile. Norma técnica: Vigilancia de Laboratorio. 2006; p. 22-5.

35.- Organización Panamericana de la Salud. Diagnóstico de laboratorio de las meningitis bacterianas causadas por Neisseria meningitidis Manual de procedimientos de laboratorio de la red SIREVA II. Sao Paulo, Brasil; 2011.

36.- Ministerio de Salud, Gobierno de Chile. Circular B51 $\mathrm{N}^{\circ} 09$. Vigilancia epidemiológica y medidas de control de enfermedad meningocócica (A39). 6 de febrero de 2009.

37.- Clinical and Laboratory Standards Institute (CLSI). Performance Standards for Antimicrobial Susceptibility Testing. TwentyTwo Informational Supplement. Vol M100-S22. ed. Wayne, PA, USA: Clinical and Laboratory Standards Institute (CLSI); 2012.

38.- Swaminathan B, Barrett T J, Hunter S B, Tauxe R V, the CDC Pulse Net Task Force. Pulse Net: The molecular subtyping network for foodborne bacterial disease surveillance, United States. 2001; Available at: http://dx.doi. org/10.3201/eid0703.017303. (Accedido en septiembre de 2013).

39.- Popovic T, Schmink S, Rosenstein N A, Ajello G W, Reeves M W, Plikaytis B, et al. Evaluation of pulsed-field gel electrophoresis in epidemiological investigations of meningococcal disease outbreaks caused by Neisseria meningitidis serogroup C. J Clin Microbiol 2001; 39 (1): 75-85.

40.- Maiden M C, Bygraves J A, Feil E, Morelli G, Russell J E, Urwin R, et al. Multilocus sequence typing: a portable approach to the identification of clones within populations of pathogenic microorganisms. Proc Natl Acad Sci USA 1998; 95 (6): 3140-5.

41.- Valenzuela M T, Moreno G, Vaquero A, Seoane M, Hormazábal J C, Bertoglia M P, et al. Emergencia de la cepa W135 causante de enfermedad meningocócica invasora en Chile 2012. Rev Med Chile 2013; 141 (8): 959-67.

42.- Chiavettal L, Chávez E, Ruzic A, Mollerach M, Requeira M. Vigilancia de Neisseria meningitidis en Argentina 1993-2005: distribución de serogrupos, serotipos y serosubtipos causantes de enfermedad invasiva. Rev Argent Microbiol 2007; 39 (1): 21-7.

43.- Sordelli N, Orlando N, Neyro S, Echave C, Procopio A, Fallo A, et al. Artritis meningococcicas primarias en pediatría. Presentación de nueve casos. Arch Argent Pediatr 2011; 109 (2): 150-9.

44.- World Health Organization, Centers for Disease Control and Prevention. Laboratory Methods for the Diagnosis of Meningitis caused by Neisseria meningitidis, Streptococcus pneumoniae and Haemophilus influenzae. WHO Manual 2011; 2nd Edition.

45.- Fasce R A, Tognarelli J, Mora J, Torres G, Andrade W, Bustos P, et al. Genetic characterization of the virus causing H1N1 influenza pandemic in Chile: analysis of the first detected cases. Rev Med Chile 2011; 139 (7): $833-40$.

46.- Chowell G, Towers S, Viboud C, Fuentes R, Sotomayor V, Simonsen L, et al. The influence of climatic conditions on the transmission dynamics of the $2009 \mathrm{~A} / \mathrm{H} 1 \mathrm{~N} 1$ influenza pandemic in Chile. BMC Infect Dis 2012; 12: 298.

47.- Ministerio de Salud. Departamento de Epidemiología. Situación Enfermedad Meningocócica por serogrupo W135. 2013; Available at: http://epi.minsal.cl/vigilanciaepidemiologica/enfermedades-de-notificacionobligatoria/enfermedad-meningococica/. (accedido el 4 de septiembre de 2013).

48.- Instituto de Salud Pública. Ministerio de Salud. Informe vigilancia de laboratorio: Enfermedad invasora Neisseria meningitidis hasta semana 41, 2013 Chile. 2013; Available at: http://www. ispch.cl/vigilancia. (accedido octubre de 2013).

49.- Safadi M A, Cintra O A. Epidemiology of meningococcal disease in Latin America: current situation and opportunities for prevention. Neurol Res 2010; 32 (3): 263-71.

50.- Ni J D, Jin Y H, Dai B, Wang X P, Liu D Q, Chen X, et al. Recent epidemiological changes in meningococcal disease may be due to the displacement of serogroup A by serogroup C in Hefei City, China. Postgrad Med J 2008; 84 (988): 87-92.

51.- Organización Panamericana de la Salud. Informe Regional de SIREVA II, 2006. Datos por país y por grupos de edad sobre las características de los aislamientos de Streptococcus pneumoniae, Haemophilus influenzae y Neisseria meningitidis en procesos invasores. Documentos técnicos. Regulaciones Sanitarias Internacionales, Alerta y Respuesta y Enfermedades Epidémicas (HSD/IR). Washington, D.C.: OPS; 2006.

52.- Organización Panamericana de la Salud. Informe Regional de SIREVA II, 2007. Datos por país y por grupos de edad sobre las características de los aislamientos de Streptococcus pneumoniae, Haemophilus influenzae y Neisseria meningitidis en procesos invasores. Documentos técnicos. Regulaciones Sanitarias Internacionales, Alerta y Respuesta y Enfermedades Epidémicas (HSD/IR). Washington, D.C.: OPS; 2007.

53.- Organización Panamericana de la Salud. Informe Regional de SIREVA II, 2008. Datos por país y por grupos de edad sobre las características de los aislamientos de Streptococcus pneumoniae, Haemophilus influenzae y Neisseria meningitidis en procesos invasores. Documentos técnicos. Regulaciones Sanitarias Internacionales, Alerta y Respuesta y Enfermedades Epidémicas (HSD/IR). Washington, D.C.: OPS; 2008.

54.- Organización Panamericana de la Salud. Informe Regional de SIREVA II, 2009. Datos por país y por grupos de edad sobre las características de los aislamientos de Streptococcus pneumoniae, Haemophilus influenzae y Neisseria meningitidis en procesos invasores. Documentos técnicos. Regulaciones Sanitarias Internacionales, Alerta y Respuesta y Enfermedades Epidémicas (HSD/IR). Washington, D.C.: OPS; 2009.

55.- Organización Panamericana de la Salud. Informe Regional de SIREVA II, 2010. Datos por país y por grupos de edad sobre las características de los aislamientos de Streptococcus pneumoniae, Haemophilus influenzae y Neisseria meningitidis en procesos 
invasores. Documentos técnicos. Regulaciones Sanitarias Internacionales, Alerta y Respuesta y Enfermedades Epidémicas (HSD/IR) Washington, D.C.: OPS; 2010.

56.- Organización Panamericana de la Salud. Informe Regional de SIREVA II, 2011. Datos por país y por grupos de edad sobre las características de los aislamientos de Streptococcus pneumoniae, Haemophilus influenzae y Neisseria meningitidis en procesos invasores. Documentos técnicos. Regulaciones Sanitarias Internacionales, Alerta y Respuesta y Enfermedades Epidémicas (HSD/IR) Washington, D.C.: OPS; 2011.

57.- Organización Panamericana de la Salud. Informe Regional de SIREVA II, 2012.

Datos por país y por grupos de edad sobre las características de los aislamientos de Streptococcus pneumoniae, Haemophilus influenzae y Neisseria meningitidis en procesos invasores. Documentos técnicos. Regulaciones Sanitarias Internacionales, Alerta y Respuesta y Enfermedades Epidémicas (HSD/IR). Washington, D.C.: OPS; 2012.

58.- Barra G N, Araya P A, Fernández J O, Gabastou J M, Hormazábal J C, Seoane M, et al. Molecular characterization of invasive Neisseria meningitidis strains isolated in Chile during 2010-2011. PLoS One 2013; 8 (6): e66006.

59.- Safadi M A, McIntosh E D. Epidemiology and prevention of meningococcal disease: a critical appraisal of vaccine policies. Expert Rev Vaccines 2011; 10 (12): 1717-30.

60.- Sorhouet-Pereira C, Efron A, Gagetti P,
Faccone D, Regueira M, Corso A, et al. Phenotypic and genotypic characteristics of Neisseria meningitidis disease-causing strains in Argentina, 2010. PLoS One 2013; 8 (3): e58065.

61.- Mayer L W, Reeves M W, Al-Hamdan N, Sacchi C T, Taha M K, Ajello G W, et al. Outbreak of W135 meningococcal disease in 2000: not emergence of a new W135 strain but clonal expansion within the electrophoretic type-37 complex. J Infect Dis 2002; 185 (11): 1596-605.

62.- Popovic T, Sacchi C T, Reeves M W, Whitney A M, Mayer L W, Noble C A, et al. Neisseria meningitidis serogroup W135 isolates associated with the ET-37 complex. Emerg Infect Dis 2000; 6 (4): 428-9. 